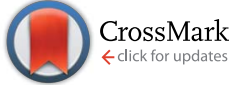

Cite this: J. Mater. Chem. A, 2015, 3, 17612

Received 22nd March 2015 Accepted 16th July 2015

DOI: $10.1039 / \mathrm{c} 5 \mathrm{ta0} 2098 \mathrm{k}$

www.rsc.org/MaterialsA

\section{Cysteine-containing oligopeptide $\beta$-sheets as redispersants for agglomerated metal nanoparticles}

\author{
Tsukasa Mizutaru, ${ }^{a}$ Taro Sakuraba, ${ }^{a}$ Toru Nakayama, ${ }^{a}$ Galina Marzun, ${ }^{b}$ \\ Philipp Wagener, ${ }^{\mathrm{b}}$ Christoph Rehbock, ${ }^{\mathrm{b}}$ Stephan Barcikowski, ${ }^{\mathrm{b}}$ Katsuhisa Murakami, ${ }^{\mathrm{cd}}$ \\ Junichi Fujita, ${ }^{\text {cd }}$ Noriyuki Ishiie and Yohei Yamamoto*ad
}

\begin{abstract}
Oligopeptide $\beta$-sheets comprising a fluorenyl methoxy carbonyl (Fmoc) group on its $\mathrm{N}$-terminus and five amino acid residues of cysteine, lysine and valine displays redispersive properties with respect to agglomerated metal nanoparticles (MNPs, M = Au, Cu, Pt and Pd). The ligand-free MNPs prepared by a laser ablation technique in liquid maintain a high dispersion state due to the inherent surface charges delivered by anionic species present in solution, but may agglomerate after the preparation depending on concentration or salinity. We show how the agglomerated MNPs can be returned to the dispersed state by adding the Fmoc-oligopeptide $\beta$-sheets in methanol, as characterized by photoabsorption spectroscopy and transmission electron microscopy. Systematic studies in which we vary the concentration, the amino acid sequences and the secondary structures of a series of the oligopeptides clarify that the $\beta$-sheet structure is essential for the redispersion of the MNPs, where metal-binding thiol groups are integrated on one side and positively charged amino groups are located on the other side of the $\beta$-sheet. A possible mechanism for the redispersion may be that the agglomerated MNPs are subsequently enwrapped by the flexible $\beta$-sheets and gradually separated due to the reconstruction of peptide $\beta$-sheets under the assembly/disassembly equilibrium.
\end{abstract}

\section{Introduction}

Metal nanoparticles (MNPs) are widely utilized as catalysts and photocatalysts, ${ }^{1}$ additives for solar cells ${ }^{2}$ and as materials for chemical and biological sensing ${ }^{3}$ due to their unusual electronic and plasmonic states. Fabrication procedures of MNPs include liquid-, gas- and solid-phase processes. ${ }^{4}$ The morphology of the MNPs is generally retained when they are immobilized on a solid surface or in a supported medium. On the other hand, agglomeration of MNPs often takes place in solution. The agglomeration suppresses their plasmonic characteristics, which is disadvantageous for utilizing MNPs as tools

${ }^{a}$ Division of Materials Science, Faculty of Pure and Applied Sciences, University of Tsukuba, 1-1-1 Tennodai, Tsukuba, Ibaraki 305-8573, Japan. E-mail: yamamoto@ ims.tsukuba.ac.jp

${ }^{b}$ Technical Chemistry I and Center for Nanointegration Duisburg-Essen (CENIDE), University of Duisburg-Essen, NanoEnergieTechnikZentrum (NETZ), Carl-BenzStrasse 199, 47057 Duisburg, Germany

'Division of Applied Physics, Faculty of Pure and Applied Sciences, University of Tsukuba, 1-1-1 Tennodai, Tsukuba, Ibaraki 305-8573, Japan

${ }^{d}$ Tsukuba Research Center for Interdisciplinary Materials Science (TIMS), Faculty of Pure and Applied Sciences, University of Tsukuba, 1-1-1 Tennodai, Tsukuba, Ibaraki 305-8573, Japan

${ }^{e}$ National Institute of Advanced Industrial Science and Technology (AIST), 1-1-1 Higashi, Tsukuba, Ibaraki 305-8566, Japan for plasmonic sensing. In addition, a well-dispersed stable colloid is a key requirement for a diverse range of other applications such as biomedicine, ${ }^{5}$ toxicology studies ${ }^{6}$ or catalysis. ${ }^{7}$

Especially, when the nanoparticles are small, the high surface energy of the particles can cause agglomeration. As the size of the particles decreases, the interaction forces between particles increases, resulting in a loss of their specific properties. Furthermore, once the agglomeration occurs, it is rather difficult to redisperse nanoparticles to their original dispersed state. $^{\mathbf{8}}$

Ligand free MNPs in aqueous or organic solvents can be produced by pulsed laser ablation in liquids. ${ }^{9}$ The irradiation with a focused high energy density laser beam of a metal target in a solvent results in the production of highly dispersed MNPs with diameters of several to several tens of nanometres. Because the resulting MNPs are charged and their surfaces are covered with anionic species that are present in solution, a repulsive electrostatic force exists between the particles. Accordingly, the MNPs maintain their highly dispersed colloidal state in solutions of micromolar salinity and at moderate particle concentrations. ${ }^{9}$ However, in pure solvents or at high concentrations, the stability of the dispersed MNPs gradually diminishes and several months after the preparation of the colloidal solution, agglomeration of the MNPs might occur. In such cases, the plasmonic characters of the MNPs are altered due to plasmon 
coupling, inducing a red shift of the surface plasmon resonance (SPR) band and an increased scattering in the near infrared (NIR) spectral regime. ${ }^{10}$ Therefore, if the agglomerated MNPs can be redispersed efficiently, the plasmonic property will be recovered to the original state, and the MNPs can be utilized as renewable materials, which are beneficial from an ecological and sustainable point of view.

Redispersion may be done via ultrasonification in a solvent, where the liquid is oscillating due to the energy transfer and causes nucleation and collapse of solvent bubbles. Then agglomerates can be broken due to the bubble collapse on the solid surfaces. Deagglomeration is then influenced by the input energy and time. However, reagglomeration can take place in case the particles are not sufficiently stabilized. Furthermore abrasion of the ultrasonic horn can occur, which may cause unwanted contaminations.

In this study, we developed redispersants for agglomerated MNPs, and found that a short oligopeptide with a certain amino acid sequence can redisperse them very efficiently. The secondary structure of the oligopeptides ( $\beta$-sheet structure) plays an important role for the redispersion of MNPs. When peptides form a $\beta$-sheet, the side chains of the amino acids protrude alternately on the upper and lower sides of the $\beta$-sheet. ${ }^{11}$ The redispersive oligopeptides include one cysteine (C) and one lysine (K) residue. Cysteine has a thiol side chain that tends to bind with metals, while lysine has an amino group on the side chain which is positively charged in a neutral aqueous solution. As a result of the $\beta$-sheet formation, metalbinding with thiol side chains predominantly occurs on one side of the $\beta$-sheet, while the positive charges are located on the other side. These bilateral properties of the peptide $\beta$-sheet may be responsible for redispersing agglomerated MNPs. A fluorenyl methoxy carbonyl (Fmoc) group attached on the N-terminus of the oligopeptides plays a role in facilitating the $\beta$-sheet formation of the peptide.

\section{Experimental}

\subsection{Synthesis and characterization of oligopeptides}

Oligopeptides are synthesized with a typical Fmoc-solid phase peptide synthesis technique using the following three protocols; [i] Fmoc deprotection, [ii] loading of Fmoc-amino acid monomer and [iii] cleavage from the resin, as reported previously. ${ }^{11}$

[i] Fmoc deprotection. Typically, piperidine $(2 \mathrm{~mL})$ was added to a dimethylformamide (DMF, $8 \mathrm{~mL}$ ) suspension of Fmoc-SAL resin ( $0.55 \mathrm{mmol} \mathrm{g}^{-1}, 0.5 \mathrm{~g}$, Watanabe Chemical Co.), and the mixture was stirred for $20 \mathrm{~min}$ at $25{ }^{\circ} \mathrm{C}$. The solution was removed by filtration and the resin was washed ten times with DMF (3 mL, 1 min stirring).

[ii] Loading of Fmoc-amino acid monomer. Typically, to the resin subjected to protocol [i] was added Fmoc-amino acid monomer (Fmoc-Lys(Trt)-OH, Fmoc-Cys(Trt)-OH, Fmoc-Val$\mathrm{OH}$, Fmoc-Glu(Trt)-OH, or Fmoc-His(Trt)-OH, $1.1 \mathrm{mmol}$ ), HBTU (375 mg, $0.99 \mathrm{mmol}$ ), HOBT (148 mg, $1.1 \mathrm{mmol})$, DMF (10 mL), and DIPEA (385 $\mu \mathrm{L}, 2.2 \mathrm{mmol})$ in this order, and the mixture was stirred for $1 \mathrm{~h}$. The solvent was removed by filtration and the resin was washed ten times with DMF. The procedures [i] and [ii] were repeated for preparation of resin bearing Fmocoligopeptides. For the preparation of H-oligopeptide, the procedure [i] was repeated one more time.

[iii] Cleavage from the resin. Typically, the resin bearing the R-oligopeptide $(\mathrm{R}=$ Fmoc or $\mathrm{H}$ ) was washed three times with $\mathrm{Et}_{2} \mathrm{O}$ (3 mL, $5 \mathrm{~min}$ stirring), and then dried under vacuum. To the resin was added a mixture of $\mathrm{CF}_{3} \mathrm{CO}_{2} \mathrm{H}$ (TFA, $2.85 \mathrm{~mL}$ ), $\mathrm{Et}_{3} \mathrm{SiH}(0.075 \mathrm{~mL})$ and $\mathrm{H}_{2} \mathrm{O}(0.075 \mathrm{~mL})$, and the mixture was stirred for $2 \mathrm{~h}$ at $25{ }^{\circ} \mathrm{C}$. The mixed solution of the resin was washed alternately with $\mathrm{MeOH}(3 \mathrm{~mL}, 3 \mathrm{~min}$ stirring) and $\mathrm{CH}_{2} \mathrm{Cl}_{2}$ (3 $\mathrm{mL}, 3 \mathrm{~min}$ stirring) three times. The solution was evaporated to dryness, and the residue was purified by reprecipitation with $\mathrm{MeOH} / \mathrm{Et}_{2} \mathrm{O}$ at least three times, affording the desired R-oligopeptide as a white powdery substance with 20$40 \%$ yield.

The products were subjected to recycling preparative HPLC (Japan Analytical Industry model LC-9210 II NEXT recycling preparative HPLC equipped with a JAIGEL-ODS-AP-A column) with $\mathrm{MeOH} / \mathrm{TFA}(100 / 0.1)$ as an eluent, where the major fraction was collected and evaporated to allow isolation of the corresponding R-oligopeptide as white solid. The final products were characterized by MALDI-TOF MS spectrometry on an AB SCIEX model TOF/TOF(TM) 5800 system spectrometer using CHCA as a matrix. The synthesized Fmoc-peptide, for example, FmocVKVVC as representative, is soluble in $\mathrm{MeOH}$, EtOH and dimethyl sulfoxide, while solubility in water, acetone and chloroform is low.

\subsection{Self-assembly of oligopeptides and characterization of the secondary structure}

A given amount of oligopeptide was dissolved in $\mathrm{MeOH}$ (0.5 mM), and the resultant solution was kept at $25^{\circ} \mathrm{C}$ for 7 days in the dark. The secondary structures were evaluated by circular dichroism (CD) spectroscopy, X-ray diffraction (XRD), attenuated total reflection (ATR) Fourier transform infrared (FT-IR) spectroscopy and transmission electron microscopy (TEM). CD spectra in $\mathrm{MeOH}(0.5 \mathrm{mM})$ were recorded at $25^{\circ} \mathrm{C}$ with a JASCO model J-1000 spectropolarimeter using a $1 \mathrm{~mm}$ path length SQ-grade quartz cell. ATR FT-IR spectra were recorded at $25{ }^{\circ} \mathrm{C}$ with a JASCO model FT/IR-4200 Fourier transform infrared spectrometer equipped with a model PR0450-S ATR attachment. For sample preparation, two drops of a $\mathrm{MeOH}$ suspension containing oligopeptide $(0.5 \mathrm{mM})$ were put onto ATR sample stage and air-dried. XRD patterns were recorded at $25{ }^{\circ} \mathrm{C}$ on a Rigaku model MiniFlex600 X-ray diffractometer with a $\mathrm{CuK} \alpha$ radiation source $\left(\lambda=1.5406 \AA\right.$, $40 \mathrm{kV}, 15 \mathrm{~mA}$, scan speed: $0.2^{\circ}$ $\min ^{-1}$ ) equipped with a model D/Tex Ultra 2-MF high-speed 1D detector. All the diffraction patterns were obtained with a $0.01^{\circ}$ step in 2 $\theta$. TEM images of self-assembled Fmoc-VKVVC were recorded on a FEI Tecnai F20 transmission electron microscope operating an accelerating voltage at $120 \mathrm{kV}$. For sample preparation, a dispersion of the self-assembled sample $(\sim 5 \mu \mathrm{L})$ was drop-cast onto a specimen grid covered with a thin carbon support film, which was hydrophilized by glow discharge. Images were recorded on a Gatan slow scan CCD camera 
(Retractable Multiscan Camera) under low dose conditions. TEM of AuNPs and those mixed with self-assembled FmocVKVVC were recorded on a JEOL model JEM-2100 transmission electron microscope operating an accelerating voltage at $200 \mathrm{kV}$. For preparation, sample dispersions were drop-cast onto a 200 mesh $\mathrm{Cu}$ grid covered with a carbon grid (Nisshin EM), which was hydrophilized by oxygen plasma for $30 \mathrm{~s}$ with $100 \mathrm{~W}$ (Yamato model PR500 plasma reactor). The sample was airdried overnight in atmosphere.

Photoluminescence (PL) spectra were measured at $25^{\circ} \mathrm{C}$ with a JASCO model FP-6500 spectrofluorometer. For sample preparation, $\mathrm{MeOH}$ solutions of tris(8-hydroxyquinoline)aluminum $\left(\mathrm{Alq}_{3}, 0.22 \mathrm{mM}, 1 \mathrm{~mL}\right)$ and its mixture with self-assembled Fmoc-VKVVC in $\mathrm{MeOH}(0.5 \mathrm{mM}$, total amount of $1 \mathrm{~mL})$ were added to aqueous solutions of AuNPs $(1 \mathrm{~mL})$. A $10 \mathrm{~mm}$ path length quartz cuvette was used for PL measurements with the excitation wavelength, excitation and emission band widths, response and sensitivity factors set at $390 \mathrm{~nm}, 3 \mathrm{~nm}, 3 \mathrm{~nm}, 0.02$ $\mathrm{s}$ and medium, respectively. The scan rate and the number of integration were $2000 \mathrm{~nm} \min ^{-1}$ and 10, respectively. In addition, a $\mathrm{MeOH} /$ water mixed solution of $\mathrm{Alq}_{3}$ was prepared and measured as a control.

For propensity calculation of the $\beta$-sheet formation, the TANGO algorithm program was utilized, which was freely downloaded from the internet at http://tango.embl.de/.12 ${ }^{12}$ The calculation was carried out under the following conditions; $\mathrm{pH}$ $=8.5, T=298.15 \mathrm{~K}$, and ionic strength $=0.004$.

\subsection{Preparation of metal nanoparticle and redispersion with peptides}

Aqueous and organic solvent dispersions of MNPs were prepared according to the reported procedure. ${ }^{9}$ Laser generation of surfactant-free metal nanoparticles was carried out using a picosecond Nd:YAG laser (Ekspla Atlantic 1064/532) with 9.8 ps pulse duration and at a repetition rate of $100 \mathrm{kHz}$. A metal target (Au 99.99\% Alfa Aesar, Cu 99.95\% Goodfellow, Pt 99.99\% Alfa Aesar and Pd 99.95\% Alfa Aesar) was placed in a self-constructed batch reactor made of Teflon..$^{9 c, 9 j}$ The reactor was filled with $30 \mathrm{~mL}$ solvent and the target was irradiated at a fundamental wavelength of $1064 \mathrm{~nm}$ with laser pulse energy of $126 \mu \mathrm{J}$. During the laser ablation, the focused laser beam was spirally scanned on the target with a scanner optic, which allows an increasing productivity due to a temporal and spatial bypassing of the cavitation bubble. The concentrations of the MNPs were determined by weighing the targets before and after ablation with a microbalance (PESA Weighing Systems). The concentration values were $55.2 \mu \mathrm{g} \mathrm{mL}^{-1}$ for AuNPs in water, $21.4 \mu \mathrm{g} \mathrm{mL}^{-1}$ for CuNPs in acetone, $47.6 \mu \mathrm{g} \mathrm{mL^{-1 }}$ for PtNPs in $0.1 \mathrm{mM} \mathrm{NaOH}$ aqueous solution and $96.7 \mu \mathrm{g} \mathrm{mL}^{-1}$ for PdNPs in $0.2 \mathrm{mM} \mathrm{NaOH}$ aqueous solution.

For redispersion experiments, $\mathrm{MeOH}$ solutions of oligopeptides $(0.5 \mathrm{mM})$, which had been incubated for 7 days, were added to the MNP dispersion $(1 / 1 \mathrm{v} / \mathrm{v})$. At this peptide concentration, the amount of added molecules per nanoparticle is about 407 , which corresponds to $\sim 1$ peptide per surface atom assuming an average particle size of $5 \mathrm{~nm}$. The mixtures were kept for 3 days in dark in order to avoid the effect of light. The redispersion experiments using a $\mathrm{MeOH}$ solution of 1-dodecanethiol $\left(\mathrm{C}_{12} \mathrm{H}_{25} \mathrm{SH}\right)(0.5 \mathrm{mM})$ were carried out in the same way. Electronic photoabsorption spectra were recorded on a JASCO model UV-650 UV-vis-NIR spectrophotometer within a wavelength range of 300-900 $\mathrm{nm}$.

\section{Results}

\subsection{Amino acid sequences and propensity for $\beta$-sheet formation}

In advance of the peptide synthesis, the propensity for $\beta$-sheet formation was simulated using the TANGO algorithm program. ${ }^{12}$ For the formation of $\beta$-sheets with thiol side chains of $\mathrm{C}$ on one side and one amino side chain of $\mathrm{K}$ on the other side, the following two conditions are adopted. [1] The total number of the amino acid residues was set to odd in order to obtain such side chain configuration for $\beta$-sheets with both parallel and antiparallel conformations. In this study, five amino acid residues (pentapeptide) were adopted. [2] One $\mathrm{C}$ and one $\mathrm{K}$ were located on odd- and even-numbered amino acid positions in the sequence, respectively, and three valines (V) occupied the remaining sites. Among the possible amino acid sequences satisfying these conditions, VKVVC showed the highest total TANGO score (66.2). We synthesized Fmocsubstituted VKVVC (Fig. 1a), and its deprotected form, HVKVVC, by the solid phase synthesis. As a control, Fmoc-KVVC, Fmoc-VVC, Fmoc-VC and Fmoc-C, where the number of amino acid residues in Fmoc-VKVVC was reduced one by one from the $\mathrm{N}$-terminus, and Fmoc-KC without $\mathrm{V}$ residues were synthesized. Fmoc-pentapeptides with different sequences, Fmoc-VVVKC, Fmoc-VVVVC (without $\mathrm{K}$ residue), Fmoc-VKVVE and FmocVKVVH (glutamic acid E, and histidine $\mathrm{H}$, instead of $\mathrm{C}$ ) were also synthesized for comparison.

\section{2. $\quad \beta$-Sheet formation properties of pentapeptides}

At first, the self-assembling features of R-VKVVC $(\mathrm{R}=$ Fmoc, $\mathrm{H}$, Fig. 1a) were investigated. Fig. 1b shows CD spectra of R-VKVVC in $\mathrm{MeOH}(0.5 \mathrm{mM})$. H-VKVVC displayed a negative ellipticity at around $200 \mathrm{~nm}$. On the other hand, the spectrum of FmocVKVVC showed negative ellipticity at $\sim 210 \mathrm{~nm}$, and the ellipticity turned to being positive at $200 \mathrm{~nm}$. This contrastive behaviour indicates that $\mathrm{H}$-VKVVC assembles randomly in $\mathrm{MeOH}$, while Fmoc-VKVVC adopts a $\beta$-sheet conformation. ${ }^{\mathbf{1 1 c , 1 3}}$ The powder XRD pattern of Fmoc-VKVVC showed strong diffraction peaks at $2 \theta=10$ and $18^{\circ}(d=8.8$ and $4.7 \AA$, respectively), which were attributed to the intersheet and interstrand distances of the peptide $\beta$-sheets (Fig. 1c, red). ${ }^{\mathbf{1 1 c}, \mathbf{1 4}}$ The FT-IR spectrum of Fmoc-VKVVC also showed evidence of $\beta$-sheet structure, where strong amide I and II bands were observed at 1630 and $1540 \mathrm{~cm}^{-1}$, respectively (Fig. $1 \mathrm{~d}$, red). ${ }^{13}$ On the other hand, the XRD and FT-IR spectra of H-VKVVC hardly showed any evidence of the $\beta$-sheet formation (Fig. 1c and $d$, black).

The TEM micrograph of Fmoc-VKVVC, self-assembled in $\mathrm{MeOH}$, displayed nanofibrillar structures with 10-30 nm width 
(a)

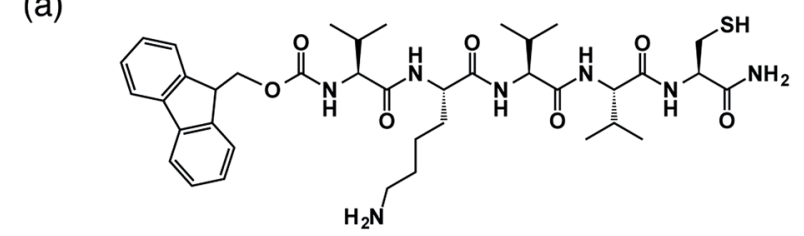

(b)

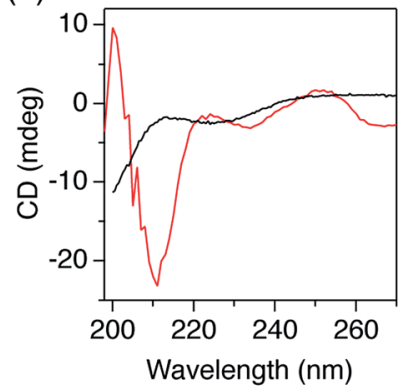

(c)

(d)
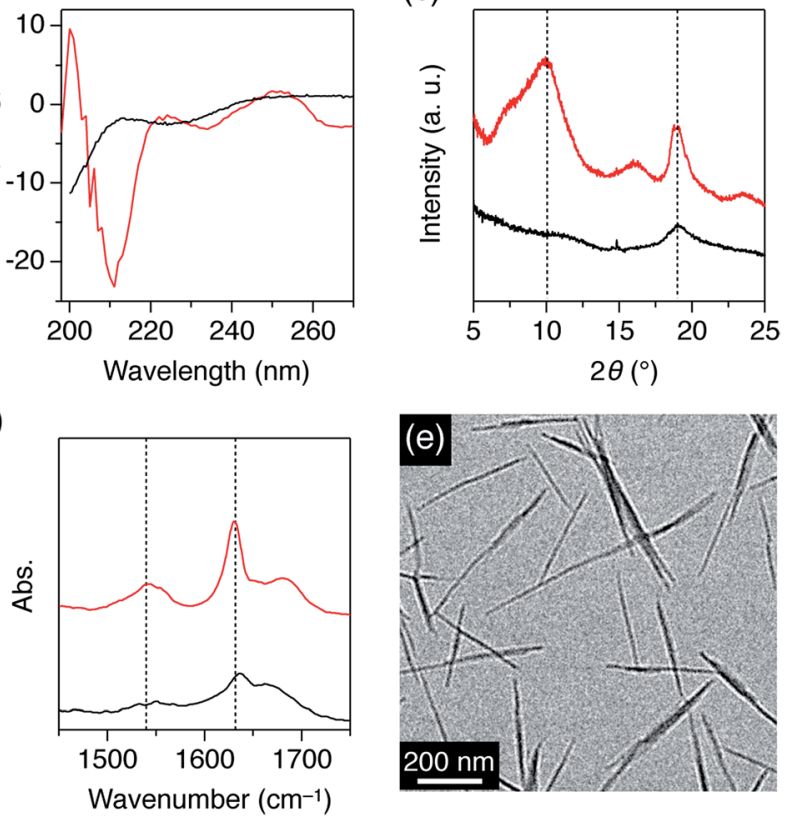

Fig. 1 (a) Molecular structure of R-VKVVC ( $R=$ Fmoc). CD spectra in $\mathrm{MeOH}(\mathrm{b}), \mathrm{XRD}$ patterns (c) and FT-IR spectra (d) of self-assembled Fmoc-VKVVC (red) and H-VKVVC (black). The dotted lines in (c) indicate $2 \theta$ angles of 10 and $18^{\circ}$, while those in (d) indicate wavenumbers of 1540 and $1630 \mathrm{~cm}^{-1}$. (e) TEM micrograph of Fmoc-VKVVC selfassembled in $\mathrm{MeOH}$.

and sub-micrometer length (Fig. 1e). Such fibrillar morphology is typical of a peptide $\beta$-sheet. Considering the amino acid sequence and previous reports on the assembly of Fmocsubstituted oligopeptides, ${ }^{15}$ the $\beta$-sheet most likely adopts an antiparallel configuration, where the side chains of $\mathrm{K}$ (animo group) and C (thiol group) are located separately on the upper and lower sides of the $\beta$-sheet, respectively.

\subsection{Redispersion of MNPs with cysteine-containing peptides}

Next, the redispersion properties of the peptides with repect to agglomerated MNPs were investigated. The as-prepared aqueous solution of highly dispersed AuNPs was coloured pink (Fig. 2a, left) due to the plasmon absorption at about $530 \mathrm{~nm}$ (Fig. 2e, dotted). However, the colour of the solution changed to blue 6 months after preparation (Fig. 2a, centre), indicating that AuNPs had become agglomerated. In fact, the TEM micrograph showed that the AuNPs were heavily agglomerated and fused with one another after 6 months (Fig. 2b).

When the MeOH solution of the $\beta$-sheets of Fmoc-VKVVC $(0.5 \mathrm{mM})$ was added to the aqueous dispersion of the agglomerated AuNPs and the mixture was aged for 3 days, the colour of the dispersion gradually turned back to pink (Fig. 2a, right), which is analogous to the initial state of the AuNP dispersion in water. Electronic photoabsorption spectroscopy showed an apparent spectral change before and after addition of the $\mathrm{MeOH}$ solution of Fmoc-VKVVC. Before addition, the absorption maximum appeared at $630 \mathrm{~nm}$ with an absorption shoulder at $530 \mathrm{~nm}$ (Fig. 2e, blue). After addition, the peak at $630 \mathrm{~nm}$ completely disappeared, and the absorption at $530 \mathrm{~nm}$ due to the surface plasmon resonance of isolated AuNPs was observed (Fig. 2e, red). TEM micrographs after addition of Fmoc-VKVVC showed that each AuNP is clearly separated from its neighbouring particle with a certain distance of 1-5 nm (Fig. 2c and d). The presence of the underlying peptides may not be detectable due to the relatively weak electron beam scattering of organic matter in comparison to heavy elements like Au. However, in the wide range view, we can identify that dispersed AuNPs are attached separately to $\beta$-sheets (Fig. 2c, inset). The pink colour of the dispersion was maintained at least 9 months after addition of the MeOH solution of Fmoc-VKVVC.

AuNPs can act as a quencher for the fluorescence of dyes. ${ }^{16}$ This phenomenon is observed in systems where the dye serves as the donor and the plasmonic NP as the acceptor species. It is predominantly attributed to two separate effects. Firstly, nonradiative energy transfer similar to FRET is found, however a second radiative effect, originating from an out of phase oscillation of fluorophores and metal NP, was also observed. ${ }^{17}$ Consequently, efficient fluorescence quenching by metal NP may also occur where the spectral overlap between fluorescence emission and NP absorbance is relatively small, e.g., in case of the combination of AuNP and red emitting dyes like Cy5. ${ }^{17 b}$ In both cases, the fluorescence quenching effect is enhanced significantly when the distance between fluorophore and NP surface is decreased as well as when the particle size is increased. ${ }^{17 b}$

We investigated the fluorescence quenching properties of the redispersed AuNPs. A MeOH/water mixed solution of $\mathrm{Alq}_{3}$ shows a PL band centered at $510 \mathrm{~nm}$ (Fig. 2f, black), which overlaps well with the plasmon absorption band of AuNPs $(\sim 530 \mathrm{~nm})$. Accordingly, fluorescence quenching by non-radiative effects is prone to occur in the observed system. In fact, $69 \%$ of the fluorescence from $\mathrm{Alq}_{3}$ was quenched when the AuNPs, redispersed by the $\beta$-sheets of Fmoc-VKVVC, were added to a solution containing $\mathrm{Alq}_{3}$ (Fig. 2f, red). The degree of quenching is much higher than in case of the original partially agglomerated AuNPs, where a quenching of $\sim 46 \%$ was observed (Fig. $2 \mathrm{f}$, blue). In comparison to literature studies, where quenching rates $>90 \%$ were observed, the values found in our study are comparably low. This is predominantly attributed to the fact that the $\mathrm{Alq}_{3}$ was not fixed on the NP surface and that the unbound dye molecules were not removed in this experimental setup. Nonetheless, a significantly more pronounced quenching was found for the dispersed particles in comparison to the aggregated ones. This may be due to the more intensive overlap between the SPR maximum of the particles and the dye's emission spectrum, which could enhance the non-radiative quenching rate. These experimental results could indicate that the plasmonic state of the redispersed AuNPs turned back to 
(a)

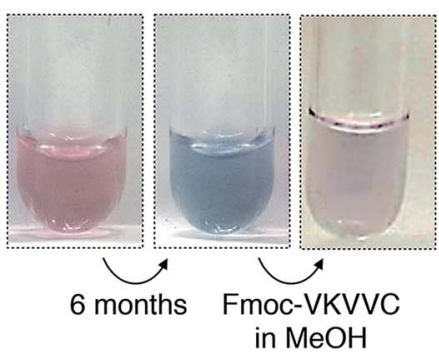

(e)

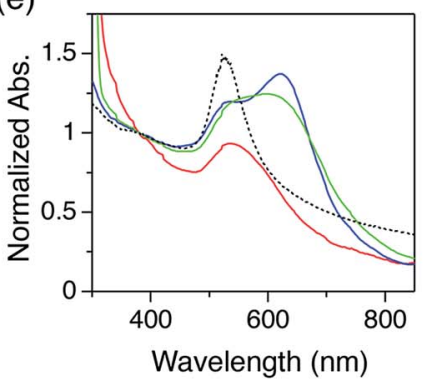

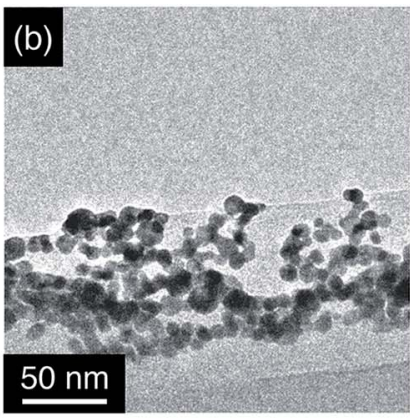

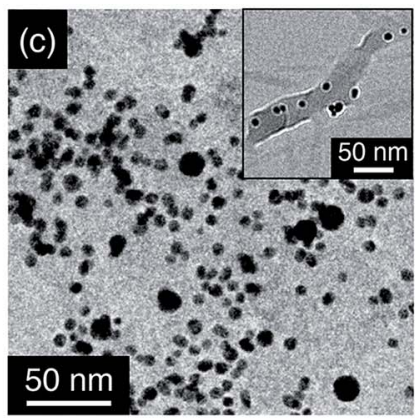

(f)

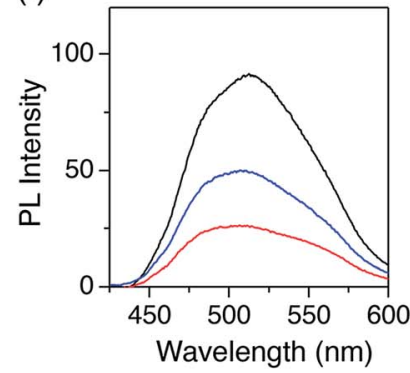

(g)

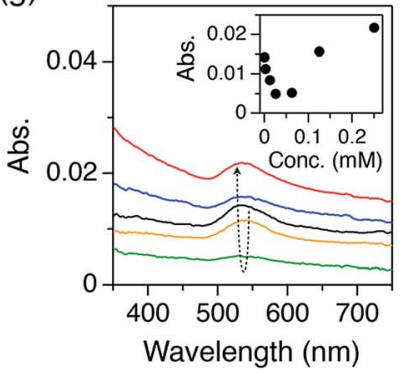

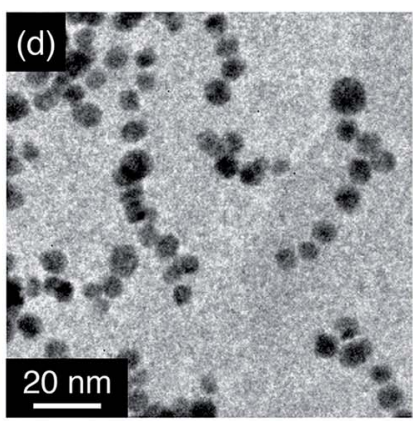

(h)

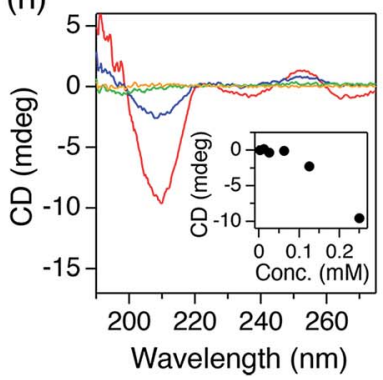

Fig. 2 (a) Photographs of the aqueous dispersion of AuNPs just after preparation (left), 6 months after preparation (centre) and finally 6 months after preparation, on the addition of a MeOH solution of self-assembled Fmoc-VKVVC and subsequent aging for 3 days (right). (b) TEM micrograph of agglomerated AuNPs in water. (c and d) TEM micrographs of agglomerated AuNPs after addition of MeOH solution containing Fmoc-VKVVC. (e) Electronic photoabsorption spectra of as-prepared AuNPs in water (dotted), agglomerated AuNPs in water (blue), and after addition of $\mathrm{MeOH}$ solution of Fmoc-C (green) and Fmoc-VKVVC (red) into the agglomerated AuNPs in water. The spectra were normalized by the interband absorption at $380 \mathrm{~nm}$, where the absorbance is not influenced by the agglomerated state but is proportional to gold mass. ${ }^{9 d}$ ( $\mathrm{f}$ ) Photoemission spectra of $\mathrm{MeOH} /$ water $(1 / 1 \mathrm{v} / \mathrm{v})$ mixed solution of $\mathrm{Alq}_{3}$ (black), mixture of $\mathrm{Alq}_{3}$ and agglomerated AuNPs (blue), and Alq 3 and AuNPs redispersed by self-assembled Fmoc-VKVVC (red). (g) Electronic photoabsorption spectra of agglomerated AuNPs (black) and that mixed with Fmoc-VKVVC in MeOH. Total concentrations of Fmoc-VKVVC in MeOH/water (1/1 v/v): 0.0025 (orange), 0.0625 (green), 0.125 (blue) and $0.25 \mathrm{mM}$ (red). Inset shows plot of absorbance at $530 \mathrm{~nm}$ versus total concentration of Fmoc-VKVVC. (h) CD spectra of Fmoc-VKVVC in MeOH/ water (1/1 v/v). Total concentrations of Fmoc-VKVVC: 0.0025 (orange), 0.0625 (green), 0.125 (blue) and 0.25 mM (red). Inset shows plot of CD intensity at $210 \mathrm{~nm}$ versus total concentration of Fmoc-VKVVC.

(a) CuNP

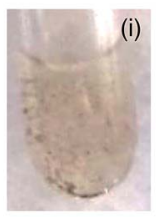

(b) PtNP

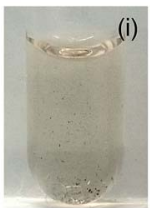

(c) PdNP
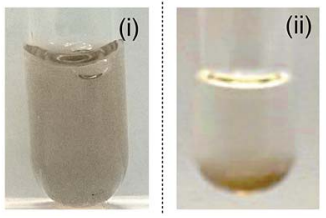

(ii)
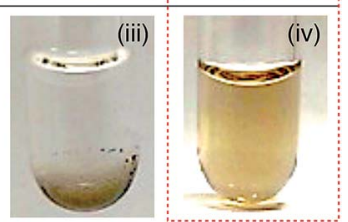

Fig. 3 Photographs of dispersions of CuNPs (a), PtNPs (b) and PdNPs (c) 6 months after preparation (i) and after addition of $\mathrm{MeOH}$ (ii), $\mathrm{MeOH}$ solution of Fmoc-C (iii) and MeOH solution of Fmoc-VKVVC (iv).

that of the initial state of the dispersed AuNPs. However, it should be noted that next to the plasmonic state, the total surface area of the AuNPs was also significantly increased in comparison to the agglomerated particles. Consequently, the higher abundance of surface area could lead to a higher number of binding events of the dye molecules to the metal surface, which could also result in a more pronounced quenching.

When the concentration of the added peptide solution was increased by two or three times, the incubation time required for the redispersion of agglomerated AuNPs was shortened to 12 days. On the other hand, when the concentration of the added peptide was reduced, a clear threshold for the redispersion of agglomerated AuNPs was observed. Fig. $2 \mathrm{~g}$ shows the absorbance change upon addition of Fmoc-VKVVC in $\mathrm{MeOH}$ to the aqueous solution of agglomerated AuNPs. Less than $0.0625 \mathrm{mM}$ of the total concentration of Fmoc-VKVVC resulted in much heavier agglomeration, leading to the decrease of the absorbance of AuNPs. This is probably attributed to the well-known charge compensation effect, previously described by Gamrad et al. for negatively-charged nanoparticles and positivelycharged peptides. ${ }^{9 g}$ At a certain concentration regime, an isoelectric point is reached where charge compensation induces impairment of the colloidal stability. This tendency is similar to the aggregation of AuNPs when cysteine was added to aqueous dispersion of AuNPs. ${ }^{18}$ In contrast, when the total concentrations of Fmoc-VKVVC were increased to 0.125 and $0.25 \mathrm{mM}$, the absorbance of AuNPs at $530 \mathrm{~nm}$ was increased (Fig. 2g, inset). 


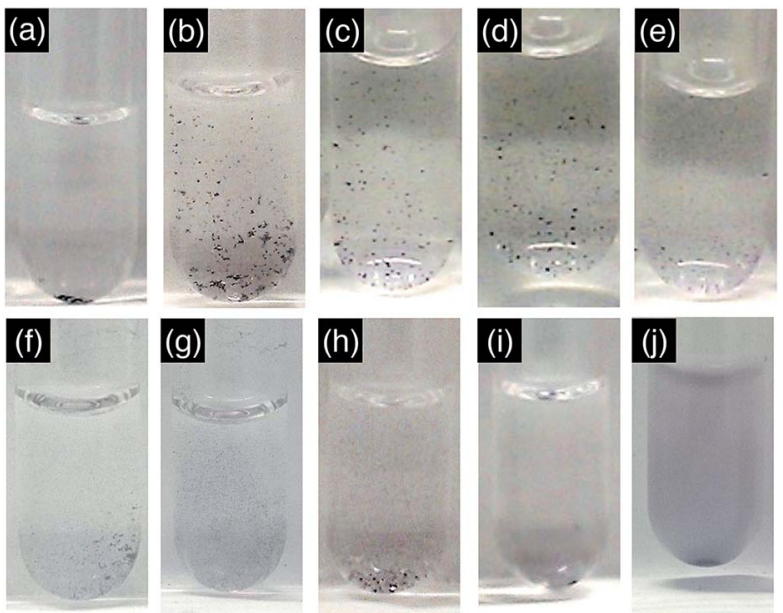

(k)

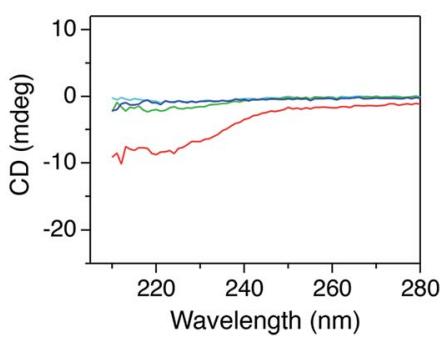

Fig. 4 Photographs of aqueous dispersions of agglomerated AuNPs after addition of $\mathrm{MeOH}$ solutions of H-VKVVC (a), Fmoc-KVVC (b), Fmoc-VVC (c), Fmoc-VC (d), Fmoc-KC (e), Fmoc-VVVKC (f), Fmoc$\operatorname{VVVVC~(g),~Fmoc-VKVVE~(h),~Fmoc-VKVVH~(i)~and~1-dodecanethiol~(j).~}$ (k) CD spectra of $\mathrm{MeOH}$ solutions of Fmoc-KVVC (red), Fmoc-VVC (blue), Fmoc-VC (green), and Fmoc-KC (light blue).

Table 1 Secondary structure in $\mathrm{MeOH}(0.5 \mathrm{mM})$ and dispersion properties of agglomerated MNPs

\begin{tabular}{lll}
\hline Sequence \& structure & Assembly in MeOH & Dispersion property $^{a}$ \\
\hline Fmoc-VKVVC & $\beta$-Sheet & $\bigcirc$ \\
H-VKVVC & Random & $\times$ \\
Fmoc-KVVC & Random & $\times$ \\
Fmoc-VVC & Random & $\times$ \\
Fmoc-VC & Random & $\times$ \\
Fmoc-C & Random & $\times$ \\
Fmoc-KC & Random & $\times$ \\
Fmoc-VVVKC & Random & $\times$ \\
Fmoc-VVVVC & Insoluble & $\times$ \\
Fmoc-VKVVE & $\beta$-Sheet & $\times$ \\
Fmoc-VKVVH & Random & $\times$ \\
1-Dodecanethiol & Soluble & $\times$ \\
${ }^{a}$ O; highly dispersive, & $\times$; hardly dispersive. ${ }^{b}$ Ref. $11 \mathrm{c.}$.
\end{tabular}

The CD spectra of Fmoc-VKVVC also show concentration dependency, where concentrations of Fmoc-VKVVC higher than $0.125 \mathrm{mM}$ display negative ellipticity at $210 \mathrm{~nm}$, while those less than $0.0625 \mathrm{mM}$ show negligible ellipticity in that wavelength region (Fig. 2h). These spectroscopic results clearly indicate that the $\beta$-sheet formation of Fmoc-VKVVC is essential for the redispersion of agglomerated AuNPs. (a)
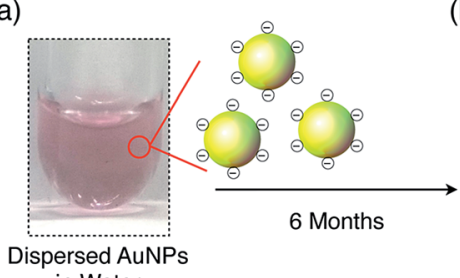

(b)

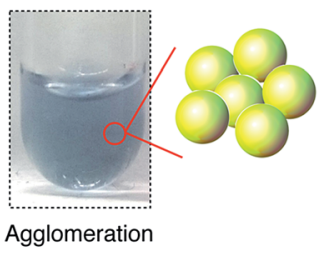

in Water
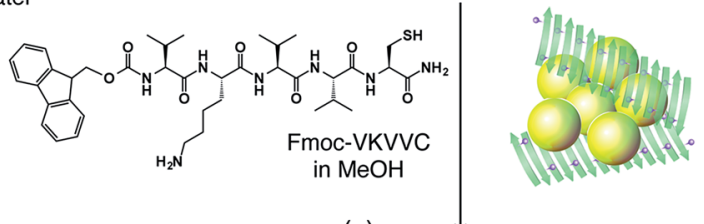

(c)

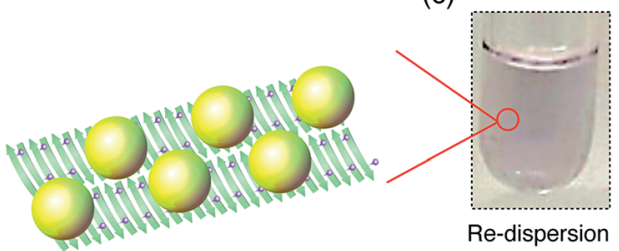

Fig. 5 Schematic representation of the redispersion of MNPs by the addition of Fmoc-VKVVC ( $\beta$-sheet) in $\mathrm{MeOH}$. Photographs in $(\mathrm{a}-\mathrm{c})$ show as-prepared AuNP dispersion in water (a), AuNP dispersion in water 6 months after preparation (b) and that added to $\mathrm{MeOH}$ solution of Fmoc-VKVVC and aged for 3 days (c). Yellow spheres, green arrows and purple spheres on the green arrows indicate AuNPs, $\beta$-strands of Fmoc-VKVVC and thiol side chains, respectively.

As a control, addition of Fmoc- $\mathrm{C}$ in $\mathrm{MeOH}$ to the agglomerated AuNP aqueous dispersion hardly showed a spectral change (Fig. 2e, green).$^{18}$ Furthermore, the addition of $\mathrm{MeOH}$ to freshlyprepared AuNPs in water did not result in significant changes in the absorption spectra. Therefore, we conclude that the redispersion phenomena are solely related to Fmoc-VKVVC.

We further investigated the redispersion properties of FmocVKVVC with respect to other agglomerated MNPs $(\mathrm{M}=\mathrm{Cu}, \mathrm{Pt}$ and Pd). Before addition of the MeOH solution of Fmoc-VKVVC, the MNPs were heavily agglomerated (Fig. 3i). Upon addition of $\mathrm{MeOH}$ only or a $\mathrm{MeOH}$ solution of Fmoc-C, the agglomeration of MNPs intensified, and precipitation could be observed by eye (Fig. 3ii and iii). In contrast, upon addition of $\mathrm{MeOH}$ solutions of Fmoc-VKVVC, the precipitates were readily dispersed by a gentle stirring, and the mixed solutions became homogeneously coloured with high transparency (Fig. 3iv).

\subsection{Influence of secondary structure and amino acid sequences for redispersing MNPs}

We systematically investigated which structural components in Fmoc-VKVVC are essential for the dispersion of agglomerated MNPs. First, a $\mathrm{MeOH}$ solution of $\mathrm{H}$-VKVVC, the deprotected form of Fmoc-VKVVC, was added to the aqueous dispersion of agglomerated AuNPs. Here, redispersion of the MNPs was hardly observed (Fig. 4a). Next, the number of amino acid residues in Fmoc-VKVVC was reduced one by one from the $\mathrm{N}$-terminus, and the $\mathrm{MeOH}$ solutions of Fmoc-peptides (FmocKVVC, Fmoc-VVC, Fmoc-VC) were added to the agglomerated AuNP aqueous dispersion. In all cases, however, redispersion 
effects were not observed (Fig. 4b-d). Furthermore, Fmoc-KC, without $\mathrm{V}$ residues, hardly redispersed the agglomerated AuNPs (Fig. 4e). According to CD spectroscopy, these peptides did not form $\beta$-sheet structures in $\mathrm{MeOH}$ (Fig. 4k). Therefore, these experiments conclusively confirm that the formation of $\beta$-sheet structures is a necessary prerequisite for the redispersion of agglomerated AuNPs.

We further checked the redispersion properties of Fmocpentapeptides with different amino acid sequences, FmocVVVKC and Fmoc-VVVVC. Fmoc-VVVKC did not form $\beta$-sheet in $\mathrm{MeOH}$, and hardly redispersed the agglomerated AuNPs (Fig. 4f). Fmoc-VVVVC itself was hardly dissolved in $\mathrm{MeOH}$ and formed a macroscopic precipitation, and the dispersion of AuNPs was significantly worsened by adding the $\mathrm{MeOH}$ suspension containing Fmoc-VVVVC (Fig. 4g).

For comparison, MeOH solutions of Fmoc-VKVVE ${ }^{9 c}$ and Fmoc-VKVVH, which contain $\mathrm{E}$ (negatively charged residue) or $\mathrm{H}$ (metal-ion binding residue) instead of $\mathrm{C}$ (metal-binding residue), were added to the agglomerated AuNP dispersion. Fmoc-VKVVE formed a $\beta$-sheet structure in $\mathrm{MeOH},{ }^{9 c}$ while Fmoc-VKVVH hardly formed $\beta$-sheet. Both of these Fmocpeptides in $\mathrm{MeOH}$ did not redisperse agglomerated AuNPs (Fig. 4h and i). Accordingly, metal-binding $\mathrm{C}$ seems to be one of the critical structural components for redispersing agglomerated MNPs. We also investigated the redispersion properties in the presence of the non-peptide compound 1-dodecanethiol, which includes a metal-binding thiol group. However, the AuNPs in water agglomerated much more severely upon the addition of a $\mathrm{MeOH}$ solution of 1-dodecanethiol, though these effects were probably increased by the poor solubility of 1dodecanethiol in aqueous environments. (Fig. 4j).

\section{Discussion}

Table 1 summarizes the redispersion abilities of the synthesized peptides and 1-dodecanethiol with respect to agglomerated MNPs, along with their secondary structures in $\mathrm{MeOH}$. Only Fmoc-VKVVC disperses agglomerated MNPs efficiently. Fmocpentapeptides without a cysteine residue hardly disperse agglomerated MNPs. The role of the Fmoc group is considered to accelerate $\beta$-sheet formation via $\pi-\pi$ interaction of the fluorenyl moieties. ${ }^{11}$ On the other hand, the shorter Fmoc-peptides, Fmoc-KVVC, Fmoc-VVC, Fmoc-VC and Fmoc-KC, as well as Fmoc-amino acid, Fmoc-C, hardly disperse the agglomerated MNPs. ${ }^{18}$ Non-peptide compounds such as 1-dodecanethiol hardly exhibited any re-dispersive property with respect to AuNPs either.

Fig. 5 shows a schematic representation of the possible mechanism for $\beta$-sheet redispersion of the agglomerated MNPs. $\beta$-Sheets of Fmoc-VKVVC possess highly integrated thiol groups on one side. When a MeOH solution of the $\beta$-sheet is added to the dispersion of the agglomerated MNPs, the $\beta$-sheet subsequently enwraps the MNPs (Fig. 5b). Dissociation of the agglomerated MNPs possibly takes place in the equilibrium of association ( $\beta$-sheet formation)/dissociation process of FmocVKVVC in solution. Once the MNPs are dissociated, they will be bound on one side of the $\beta$-sheet (Fig. 5 c). The $\beta$-sheet of Fmoc-
VKVVC is dispersive in aqueous solution because of the positive charges of $\mathrm{K}$ on the other side of the $\beta$-sheet, resulting in the highly dispersed MNPs as a hybrid material. In contrast, FmocVVVKC hardly redispersed agglomerated MNPs, possibly because of the lack of the $\beta$-sheet secondary structure.

\section{Conclusions}

In this study, we found that a fluorenyl methoxycarbonyl (Fmoc)-substituted pentapeptide comprising cysteine, lysine and valine has a dispersive property with respect to agglomerated ligand-free metal nanoparticles (MNPs, $\mathrm{M}=\mathrm{Au}, \mathrm{Cu}, \mathrm{Pt}$ and Pd). Due to the potent assembling feature of Fmoc groups, the Fmoc-pentapeptide adopts a $\beta$-sheet conformation in $\mathrm{MeOH}$, thus the thiol and amino groups of cysteine and lysine, respectively, protrude and integrate separately on the upper and lower sides of the $\beta$-sheet. When the $\beta$-sheets in $\mathrm{MeOH}$ were added to the dispersion of the agglomerated MNPs and kept for several days, the colour of the dispersion turned back to the initial state of the highly dispersed MNPs. This behaviour indicates that the plasmonic state is recovered to that of the asprepared dispersed AuNPs. Systematic studies using various peptides and their concentration dependency clarified that $\beta$ sheet formation of the Fmoc-peptide is essential for the redispersion of the agglomerated MNPs. A possible redispersion mechanism is probably that the agglomerated MNPs are subsequently enwrapped by the $\beta$-sheets and gradually separated. Flexible and dispersive $\beta$-sheets play an important role for the redispersion of the agglomerated MNPs, in which reconstruction of the $\beta$-sheet structure, accompanied by the association/dissociation equilibrium of the Fmoc-pentapeptides, most likely separates off each MNP. Such redispersion of agglomerated MNPs will be useful from an ecological point of view for reusing degenerated MNPs as renewable materials.

\section{Acknowledgements}

The authors thank Prof. Kentaro Shiraki and Mr Shunsuke Yoshizawa of University of Tsukuba for help of PL measurements. This work was partly supported by KAKENHI (25107507, $15 \mathrm{H} 00860$ and 15K13812) JSPS/MEXT Japan, Asahi Glass Foundation and German Academic Exchange Service (DAAD)Tsukuba partnership program. PW and GM gratefully acknowledge funding by German Ministry of Research and Education (BMBF, FKZ 03X5523).

\section{Notes and references}

1 (a) M. Haruta, Chem. Rec., 2003, 3, 75; (b) M.-C. Daniel and D. Astruc, Chem. Rev., 2004, 104, 293; (c) E. Yoo, T. Okata, T. Akita, M. Kohyama, J. Nakamura and I. Honma, Nano Lett., 2009, 9, 2255; (d) I. X. Green, W. Tang, M. Neurock and J. T. Yates Jr, Science, 2011, 333, 736; (e) H. Yoshida, Y. Kuwauchi, J. R. Jinschek, K. Sun, S. Tanaka, M. Kohyama, S. Shimada, M. Haruta and S. Takeda, Science, 2012, 335, 317; (f) K. Ueno and H. Misawa, NPG 
Asia Mater., 2013, 5, e61; (g) F. Pincella, K. Isozaki and K. Miki, Light: Sci. Appl., 2014, 3, e133.

2 (a) J. H. Lee, J. H. Park, J. S. Kim, D. Y. Lee and K. Cho, Org. Electrochem., 2009, 10, 416; (b) F.-C. Chen, J.-L. Wu, C.-L. Lee, Y. Hong, C.-H. Kuo and M. H. Huang, Appl. Phys. Lett., 2009, 95, 013305; (c) J.-L. Wu, F.-C. Chen, Y.-S. Hsiao, F.-C. Chien, P. Chen, C.-H. Kuo, M. H. Huang and C.-S. Hsu, ACS Nano, 2011, 2, 959; (d) Y. Takahashi and T. Tatsuma, Appl. Phys. Lett., 2011, 99, 182110; (e) X. Li, W. C. Choy, H. Lu, W. E. I. Sha and A. H. P. Ho, Adv. Funct. Mater., 2013, 23, 2728; (f) S.-W. Baek, J. Noh, S.-H. Lee, B. Kim, M.-K. Seo and J.-Y. Lee, Sci. Rep., 2013, 3, 1726; (g) T. Sasaki, K. Tabata, K. Tsukagoshi, A. Beckel, A. Lorke and Y. Yamamoto, Thin Solid Films, 2014, 562, 467.

3 J. N. Anker, W. P. Hall, O. Lyandes, N. C. Shah, J. Zhao and R. P. van Duyne, Nat. Mater., 2008, 7, 442.

4 (a) F. E. Kruis, H. Fissan and A. Peled, J. Aerosol Sci., 1998, 29, 511; (b) P. Raveendran, J. Fu and S. L. Wallen, J. Am. Chem. Soc., 2003, 125, 13940; (c) T. K. Sau and C. J. Murphy, J. Am. Chem. Soc., 2004, 126, 8648; (d) W.-H. Chiang and R. M. Sankaran, Appl. Phys. Lett., 2007, 91, 121503; (e) M. Faraji, Y. Yamini, A. Saleh, M. Rezaee, M. Ghambarian and R. Hassani, Anal. Chim. Acta, 2010, 659, 172.

5 (a) W. J. Stark, Angew. Chem., Int. Ed., 2012, 50, 1242; (b) M. C. Buford, R. F. Hamilton and A. Holian, Part. Fibre Toxicol., 2007, 4, 6.

6 (a) L. K. Limbach, Y. Li, R. N. Grass, T. J. Brunner, M. A. Hintermann, M. Muller, D. Gunther and W. J. Stark, Environ. Sci. Technol., 2005, 39, 9370; (b) U. Taylor, C. Rehbock, C. Streich, D. Rath and S. Barcikowski, Nanomedicine, 2014, 9, 1971; (c) L. Foucaud, M. R. Wilson, D. M. Brown and V. Stone, Toxicol. Lett., 2007, 174, 1.

7 F. Maillard, S. Schreier, M. Hanzlik, E. R. Savinova, S. Weinkauf and U. Stimming, Phys. Chem. Chem. Phys, 2005, 7, 385.

8 V. Amendola and M. Meneghetti, Phys. Chem. Chem. Phys., 2009, 11, 3805.

9 (a) S. Petersen, A. Barchanski, U. Taylor, S. Klein, D. Rath and S. Barcikowski, J. Phys. Chem. C, 2011, 115, 5152; (b) S. Ibrahimkutty, P. Wagener, A. Menzel, A. Plech and S. Barcikowski, Appl. Phys. Lett., 2012, 101, 103104; (c) P. Nachev, D. D. van't Zand, V. Coger, P. Wagener, K. Reimer, P. M. Vogt, S. Barcikowski and A. Pich, J. Laser Appl., 2012, 24, 042012; (d) C. Rehbock, V. Merk, L. Gamrad, R. Streubel and S. Barcikowski, Phys. Chem. Chem. Phys., 2013, 15, 3057; (e) M. Lau, I. Haxhiaj, P. Wagener, R. Intartaglia, F. Brandi, J. Nakamura and S. Barcikowski, Chem. Phys. Lett., 2014, 610-611, 256; $(f)$ G. Marzun, C. Streich, S. Jendrej, S. Barcikowski and
P. Wagener, Langmuir, 2014, 30, 11928; (g) L. Gamrad, C. Rehbock, J. Krawinkel, B. Tumursukh, A. Heisterkamp and S. Barcikowski, J. Phys. Chem. C, 2014, 118, 10302; (h) V. Merk, C. Rehbock, F. Becker, U. Hagemann, H. Nienhaus and S. Barcikowski, Langmuir, 2014, 30, 4213; (i) C. Rehbock, J. Jakobi, L. Gamrad, S. van der Meer, D. Tiedemann, U. Taylor, W. Kues, D. Rath and S. Barcikowski, Beilstein J. Nanotechnol., 2014, 5, 1523; (j) G. Marzun, J. Nakamura, X. Zhang, S. Barcikowski and P. Wagener, Appl. Surf. Sci., 2015, 348, 75.

10 F. McKenzie, K. Faulds and D. Graham, Chem. Commun., 2008, 2367.

11 (a) P. Vairaprakash, H. Ueki, K. Tashiro and O. M. Yaghi, J. Am. Chem. Soc., 2011, 133, 759; (b) A. M. Fracaroli, K. Tashiro and O. M. Yaghi, Inorg. Chem., 2012, 51, 6437; (c) T. Nakayama, T. Sakuraba, S. Tomita, A. Kaneko, E. Takai, K. Shiraki, K. Tashiro, N. Ishii, Y. Hasegawa, Y. Yamada, R. Kumai and Y. Yamamoto, Asian J. Org. Chem., 2014, 3, 1182.

12 A.-M. Fernandez-Escamilla, F. Rousseau, J. Schmkowitz and L. Serrano, Nat. Biotechnol., 2004, 22, 1302.

13 E. L. Bakota, O. Sensoy, B. Ozgur, M. Sayar and J. D. Hartgerink, Biomacromolecules, 2013, 14, 1370.

14 C. J. Bowerman and B. L. Nilson, Pept. Sci., 2012, 98, 169. 15 (a) Z. Yang, H. Gu, D. Fu, P. Gao, J. K. Lam and B. Xu, Adv. Mater., 2004, 16, 1440; (b) Z. Yang, H. Gu, Y. Zhang, L. Wang and B. Xu, Chem. Commun., 2004, 208; (c) A. Mahler, M. Reches, M. Rechter, S. Cohen and E. Gazit, Adv. Mater., 2006, 18, 1365; (d) A. R. Hirst, S. Roy, M. Arora, A. K. Das, N. Hodson, P. Murray, S. Marshall, N. Javid, J. Sefcik, J. Boekhoven, J. H. van Esch, S. Santabarbara, N. T. Hunt and R. V. Ulijn, Nat. Chem., 2010, 2, 1089.

16 (a) C. Fan, S. Wang, J. W. Hong, G. C. Bazan, K. W. Plaxco and A. J. Heeger, Proc. Natl. Acad. Sci. U. S. A., 2003, 100, 6297; (b) U. S. Raikar, V. B. Tangod, B. M. Mastiholi and V. J. Fulari, Opt. Commun., 2011, 284, 4761; (c) R. Schreiber, J. Do, E.-M. Roller, T. Zhang, V. J. Schüller, P. C. Nickels, J. Feldmann and T. Liedl, Nature Nanotechnol., 2014, 9, 74; (d) D. Ghosh and N. Chattopadhyay, J. Lumin., 2015, 160, 223.

17 (a) E. Dulkeith, A. C. Morteani, T. Niedereichholz, T. A. Klar, J. Feldmann, S. A. Levi, F. van Veggel, D. N. Reinhoudt, M. Möller and D. I. Gittins, Phys. Rev. Lett., 2002, 89, 203002; (b) E. Dulkeith, M. Ringler, T. A. Klar and J. Feldmann, Nano Lett., 2005, 5, 585.

18 R. G. Acres, V. Feyer, N. Tsud, E. Carlino and K. C. Prince, J. Phys. Chem. C, 2014, 118, 10481. 